\title{
Surgery of vestibular schwannomas: An institutional experience
}

\author{
Vijendra K. Jain, Naveen Mehrotra, Rabi Narayan Sahu, Sanjay Behari, Deepu Banerji, \\ Devendra K. Chhabra
}

Department of Neurosurgery, Sanjay Gandhi Postgraduate Institute of Medical Sciences, Lucknow, India

\begin{abstract}
Aims: To report management results of vestibular schwannomas (VS) treated surgically in our institute, with particular reference to completeness of tumor excision, facial nerve and hearing preservation and complications of surgery. Study Design and Settings: Retrospective study of 259 patients treated during the years 1988 to 2002 . Materials and Methods: The facial nerve function and hearing assessment was done according to House-Brackmann [HB] grading and pure tone audiometry (PTA) respectively. All patients were operated by retro-mastoid sub-occipital approach. Results: Most patients had large tumors and had no useful hearing $(90 \%)$, had disabling cerebellar ataxia $(88 \%)$ and presented with features of raised intra-cranial pressure (45\%). Large sized tumors were in $41.3 \%$ and giant sized tumors were in $56 \%$ cases. Complete tumor excision was carried out in $96.5 \%$ and anatomical preservation of facial nerve was achieved in $79.2 \%$ cases. Hearing preservation was achieved in 8 patients. Cerebrospinal fluid leak with or without meningitis and transient lower cranial nerve paresis were common complications. The mortality was $6 \%$. Conclusions: With experience, complete tumor excision with good facial nerve preservation can be achieved in large tumors. Hearing preservation is difficult in bigger tumors. Prevention and control of infection was a major concern.
\end{abstract}

Key Words: Vestibular Schwannomas, cerebello-pontine angle, retro-mastoid approach, facial nerve preservation

\section{Introduction}

There are only a few reports discussing the management consideration in vestibular schwannomas (VS) from India. ${ }^{[1-}$ ${ }^{4]}$ To quote Ramamurthi, saving life and functions of the lower cranial nerves still forms the main goal in majority of patients who come with large tumors. ${ }^{[5]}$ We report the results of management of vestibular schwannomas in our institute.

\section{Materials and Methods}

Two hundred and sixty-five patients with VS were treated in our Institute from 1988 to December 2002. Six cases were excluded from the study as in three elderly patients only a ventriculo-peritoneal shunt was performed and three patients who had tumor recurrence after surgery in another hospital. Thus, 259 patients included in this study, had their surgery done for the first time at our institute. In 9 patients complete excision of the tumor was not achieved, as part of the tumor capsule was adherent firmly, either to the brainstem or to the facial nerve and could not be safely dissected. Thus the surgical outcome of complete excision of VS was studied in 250 patients.

The clinical records of the patients were reviewed; the facial nerve function was graded according to House-Brackmann $[\mathrm{HB}]^{[6]}$ score pre-operatively, post-operatively, at the time of discharge and at follow up. Pre-operative pure tone audiometry was done in all patients to assess the degree of hearing loss. Criteria for useful hearing was taken as hearing loss $<60$ decibel (Norstadt Classification for audiometric hearing). ${ }^{[7]}$ Post-operative hearing assessment was done only in those patients who had useful hearing preoperatively. The speech discrimination test was not done.

The tumor size was measured (in CT and/or MRI scan) in three axes that is diameter parallel to the petrous ridge, perpendicular to the petrous ridge or the vertical diameter in the coronal slices. The size of the VS was taken as the largest diameter in any one of these three axes. The tumors were then categorized according to the classification proposed by Jackler ${ }^{[8]}$ et al.

For patients with bilateral tumors, the side operated was considered for categorization.

All the patients were operated via the retro-mastoid sub-occipital transmeatal approach in park-bench position, except for two patients who were operated in sitting position. Cavitron ultrasonic aspirator was used in a few cases. The facial nerve stimulator was not utilized. The intrameatal component of the tumor was removed and the lateral aspect of the intrameatal facial nerve defined, after drilling the roof of internal acoustic meatus (LAM). A piece of muscle was used to seal the drilled IAM in all patients. In the 3 patients during reexploration for the persistent cerebrospinal fluid (CSF) rhinorrhea, fibrin glue with muscle patch was used to seal the drilled IAM. 
All the patients were followed up at 6 weeks, at 3 months, at 6 months and yearly thereafter. The maximum follow up was of 11 years.

\section{Results}

There were 151 males and 108 females with ages ranging from 11 to 78 years. No predilection was observed as far as side or sex were concerned. Eleven patients $(4.2 \%)$ had bilateral tumors (Neurofibromatosis type-2). In one of these patients bilateral surgery was performed and complete excision of the tumor was achieved on the side with the larger tumor and a sub-total excision was done on the contralateral side after a period of time. Two of these patients had coexisting small neurofibroma arising from lower cranial nerves that was asymptomatic and was detected during surgery. These tumors were not resected.

There was no useful hearing in approximately $90 \%$ patients. Varying degrees of facial nerve paresis was observed in $65.2 \%$ cases. The other symptoms were of raised intra-cranial pressure, trigeminal dysfunction and cerebellar ataxia (Table 1).

One hundred and forty five patients (56\%) had giant, $41.3 \%$ had large and $2.7 \%$ of patients had medium sized tumors (Figure 1).

Twenty-two patients underwent ventriculo-peritoneal shunt procedure prior to the definitive surgery. Thirteen (5.2\%) patients required ventriculo-peritoneal shunt surgery in the post-operative period.

Complete excision of the VS along with intra-canalicular (intra-meatal) portion was achieved in $96.5 \%$ (250/259) of patients. Staged tumor resection was done in 5 patients. Out of the nine patients in whom complete excision could not be

Table 1: Pre-operative cranial nerve and neurological deficits in patients with vestibular schwannoma

\begin{tabular}{lcc}
\hline Neurological deficits & Number & (\%) \\
Hearing loss (no useful hearing) & 233 & 89.9 \\
Cerebellar signs & 229 & 88.4 \\
Trigeminal dysfunction & 180 & 69.4 \\
Facial nerve paresis & 169 & 65.2 \\
Papilledema & 116 & 44.7 \\
Secondary optic atrophy & 36 & 5.0 \\
Lower cranial nerve paresis & 89 & 34.3 \\
\hline
\end{tabular}

Tumor Distribution (Vestibular Schwannoma)

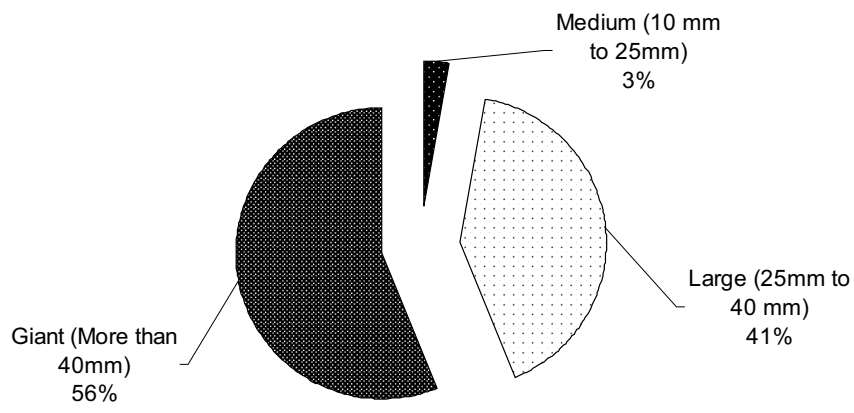

Figure 1: Size distribution of vestibular schwannomas achieved; 3 patients had tumor adhered to the brainstem and in 5 patients the tumor was adhered to the facial nerve (part of the tumor was left to avoid nerve injury). In one patient, only a subtotal removal was possible due to massive intraoperative hemorrhage.

Facial nerve was anatomically preserved in 79.2\% (198/250) patients with complete tumor excision. Nine patients died in post-operative period, and 189 patients were analyzed for facial nerve function at time of discharge. The facial nerve preservation rates were $76.5 \%$ for giant size VS and increased rates were observed with tumors of smaller sizes $(81.3 \%$ for large and $100 \%$ for medium size) (Table 2). Further, the functional status of the facial nerve at follow up were better in patients with relatively smaller tumors who had mild grade facial paresis (H\&B Grade 1 and 2) pre-operatively (Table 3 ).

Twenty-seven patients had useful hearing preoperatively. Useful hearing could be retained in 8 patients $(29.6 \%)$ postoperatively. Amongst these 8 patients, 1 had giant size tumor, 4 had large tumors and 3 had medium sized tumor.

Cerebrospinal fluid (CSF) leak occurred in 4\% (10/250) patients. CSF rhinorrrhea and CSF otorrhea occurred in 6 and 2 patients respectively. Most cases were managed conservatively with or without lumbar puncture and CSF drainage. Three patients required re-exploration and plugging of the drilled internal auditory meatus (IAM) for CSF leak. Three patients $(1.2 \%)$ developed operative site hematoma and 3 patients had an extra-dural hematoma $(1.2 \%)$ and all of them underwent re-exploration surgery. Twenty patients $(8.0 \%)$ developed post-operative meningitis. Eighteen patients responded to the anti-meningitic treatment. Vertebral artery injury occurred in $2(0.8 \%)$ patients; the procedure was abandoned and one patient underwent tumor excision at a later date. Both these patients were discharged without any neurological deficit due to vertebral artery injury. Post-operative lower cranial nerve paresis developed in 17 (6.8\%) patients (Table 4). These patients required nasogastric tube feeding and three of them required temporary tracheostomy. However the lower cranial nerve paresis improved functionally during 4-6 weeks follow-up.

The mortality was in $6 \%$ patients (15/250). Massive cerebellar edema leading to brainstem failure occurred in 3 patients. Air embolism (which includes one case operated by sitting position) and meningitis were responsible for mortality in 2 patients each. Massive intra-operative haemorrhage, operative site haematoma, post-operative myocardial infarction, fulminant septicemia and severe anaphylactic reaction due to

Table 2: Anatomical preservation of facial nerve in patients with complete excision in different tumor sizes

\begin{tabular}{lccc}
\hline Tumor & $\begin{array}{c}\text { Patients with } \\
\text { category }\end{array}$ & $\begin{array}{c}\text { Anatomical } \\
\text { complete removal }\end{array}$ & (\%) \\
pedeservation of $7^{\text {th }}$ nerve & \\
Large & 7 & 7 & 100 \\
Giant & 101 & 83 & 81.3 \\
Total & 142 & 108 & 76.5 \\
\hline
\end{tabular}


Table 3: Pre-operative and post-operative functional grading of $7^{\text {th }}$ nerve in tumors with total resection and anatomical preservation of $7^{\text {th }}$ nerve $(n=189)$

\begin{tabular}{|c|c|c|c|c|c|c|c|c|c|}
\hline \multirow[t]{2}{*}{ Grade of $7^{\text {th }}$ nerve palsy } & \multicolumn{3}{|c|}{ Giant } & \multicolumn{3}{|c|}{ Large } & \multicolumn{3}{|c|}{ Medium } \\
\hline & $\begin{array}{l}\text { Preop.* } \\
\text { [n=103] }\end{array}$ & $\begin{array}{c}\text { Disc.\# } \\
{[n=103]}\end{array}$ & $\begin{array}{l}\text { Fup. }^{\$} \\
{[\mathrm{n}=69]}\end{array}$ & $\begin{array}{l}\text { Preop. } \\
{[n=80]}\end{array}$ & $\begin{array}{c}\text { Disc. } \\
{[n=80]}\end{array}$ & $\begin{array}{c}\text { Fup. } \\
{[n=67]}\end{array}$ & $\begin{array}{l}\text { Preop } \\
{[n=6]}\end{array}$ & $\begin{array}{l}\text { Disc. } \\
{[n=6]}\end{array}$ & $\begin{array}{l}\text { Fup. } \\
{[n=2]}\end{array}$ \\
\hline Normal/Mildparesis (Grade 1 and 2) & 92 & 13 & 21 & 74 & 20 & 31 & 6 & 4 & 2 \\
\hline Moderate paresis (Grade 3 and 4 ) & 11 & 73 & 36 & 6 & 55 & 28 & 0 & 2 & 0 \\
\hline Severe paresis/palsy (Grade 5 and 6 ) & 0 & 17 & 12 & 0 & 5 & 8 & 0 & 0 & 0 \\
\hline Total & 103 & 103 & 69 & 80 & 80 & 67 & 6 & 6 & 2 \\
\hline
\end{tabular}

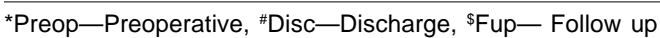

\begin{tabular}{lcc}
\hline \multicolumn{3}{c}{ Table 4: Post-operative complications in patients with com- } \\
plete excision and anatomical preservation of facial nerve \\
\hline Complications & No. of patients & $\%$ \\
CSF Leak & 10 & 4.0 \\
Hematoma & 06 & 2.4 \\
Meningitis & 21 & 8.0 \\
Vascular injury & 02 & 0.8 \\
Lower cranial nerve paresis & 17 & 6.8 \\
\hline
\end{tabular}

mismatched blood transfusion caused mortality in one patient each. No definite cause of death could be established in three patients who could not be revived following surgery.

\section{Discussion}

The last century has witnessed a considerable evolution in the management of VS with contributions from eminent neurosurgeons like Cushing who was the first to reduce mortality rates from $50 \%$ to $11 \%{ }^{[9]}$. Later Walter Dandy reported complete excision with no mortality in his series of 5 patients. ${ }^{[9]}$ In 1964, House ${ }^{[10]}$ introduced operating microscope for translabyrinthine approach and a year later in 1965 Rand and Kurze $^{[11]}$ utilized the operating microscope for trans-meatal posterior fossa approach. With the availability of operating microscope, safe modern anesthesia and refinements in the microsurgical technique the goal of VS surgery has shifted from complete excision to excellent facial nerve function and preservation of cochlear nerve function. During the past quarter of century the advances in neuro-imaging like high resolution computed tomography and multiplanar magnetic resonance imaging have resulted in early detection of VS and an increased frequency of smaller lesions being operated.

In this present study, $97 \%$ of patients had either a large or giant sized VS. Ninety percent of patients had no useful hearing at the time of presentation. In contrast to certain western literatures, ${ }^{[12,13]}$ majority of our patients sought medical attention at a stage when they developed disabling cerebellar ataxia $(88.4 \%)$ and/or the symptoms of raised intra-cranial pressure.

Pre-operative ventriculo-peritoneal shunt is not required in majority of the patients, but some patients report late with signs of raised intra-cranial pressure requiring emergency shunt procedures. In the present study the pre-operative shunt was done in 8 patients at our center and 14 patients had shunt done elsewhere before they were referred for definitive sur- gery. The incidence of preoperative shunt was as high as $66 \%$ in the series reported by Ramamurthi et al. ${ }^{[5]}$

Complete tumor excision was achieved in 96.5\% (250/259) of the patients. Yamakani et al, ${ }^{[14]}$ reported complete tumor excision in $86 \%$ patients by retro-mastoid approach for large acoustic tumors. Lanman et al reported a higher rate (96.3\%) of total removal by the trans-labrynthine approach. ${ }^{[13]}$ Ebersold et $\mathrm{al}^{[15]}$ achieved total tumor resection in $97.2 \%$ (249/256) by retro-mastoid approach for tumors of all sizes. Samii et al ${ }^{[16]}$ have reported complete excision in $97.9 \%$ patients by suboccipital trans-meatal approach.

Anatomical preservation of facial nerve was achieved in $79.2 \%(198 / 250)$ patients. In the giant category facial nerve preservation was $76.5 \%(108 / 141)$ and in the large category was $81.3 \%$ (83/102) and $100 \%$ (7/7) for medium size tumors. In some western literatures the anatomical preservation rate is $80-90 \%$ with the removal of large tumors either by the translybrinthine approach ${ }^{[13,17,18]}$ or by the retro-mastoid approach. ${ }^{[12,16,19]}$ Other authors have reported the anatomical preservation of the facial nerve for tumors $\geq 4 \mathrm{cms}$ ranging from $89 \%$ to $92 \%$ by trans-labyrinthine approach ${ }^{[20-23]}$ and $78 \%-94 \%$ by sub-occipital-retro-sigmoid approach ${ }^{[19,23]}$ Samii and Matthias reported preservation rate of $87 \%$ with tumor size $>3 \mathrm{~cm}$ until 1988, but in most recent 200 cases preservation rates rose to $94 \%$ independent of tumor size. ${ }^{[23]}$ Moreover in our series after 1995 (in recent 166 cases) we have achieved facial nerve preservation rate of $84.3 \%$ observing that experience results in better outcome. The preservation rate was 69\% (58/84) until 1995 and had subsequently increased to $84.3 \%$ (140/166) (Figure 2). This data confirms that there is a learning curve for surgery of VS. According to Whittaker et al a surgeon operating less than 12 cases per year cannot expect to get equal results of large series. ${ }^{[24]}$ The size of the tumor, is one of the important factors responsible for the preservation of the facial nerve, having a reciprocal relationship i.e. larger the size of tumor lesser the chances of preservations ${ }^{[9,13,19]}$ was also observed in this present study.

In the present study $10.4 \%$ (27/259) patients had pre-operatively useful hearing. Post-operative hearing could be preserved in 8 of these 27 patients $(29.6 \%)$ in spite of their giant size in 1, large in 4 and medium in 3 patients. Though the retro-mastoid approach gives the surgeon great opportunity for saving hearing in small sized tumors, but in tumors more than $4 \mathrm{~cm}$, the post-operative hearing is usually very poor as 


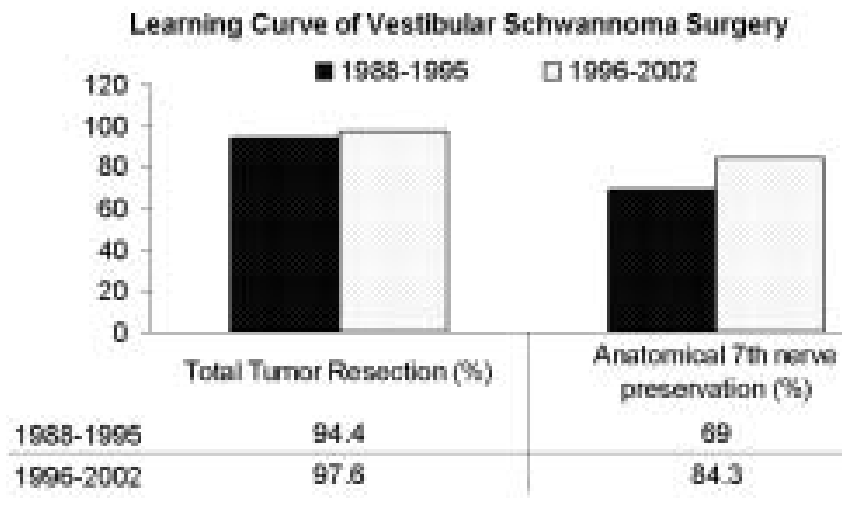

Figure 2: Anatomical preservation of facial nerve in patients with complete excision showing learning curve

observed by Ebersold et al, ${ }^{[19]}$ who reported no post-operative hearing in any of patients with tumor size more than $4 \mathrm{~cm}$. According to Samii et al, patients with large tumor (30 $\mathrm{mm} \mathrm{x}$ $20 \mathrm{~mm}$ ) hearing were preserved in $23.6 \%$ (78/330). ${ }^{[7]}$ Almost all authors agree that hearing preservation is more likely with smaller tumors with good pre-operative hearing. ${ }^{\text {25-31] }}$ Whittaker and Luetje are of the opinion that hearing preservation attempts should be made for tumors below $2 \mathrm{~cm}$ in diameter with the patient fully informed. By fully informed means that the patient understands that some hearing may be preserved in 30\%-50\% of cases (depending on tumor size), and that $30 \%$ of this number will have unexplained deterioration later, leaving some permanent hearing impairment; and probably only $7 \%$ to $9 \%$ of the total group will find hearing useful. ${ }^{[24]}$ This delayed worsening of preserved hearing was observed by Goel et al. ${ }^{[32]}$

The reported incidence of cerebrospinal fluid leak ranges between $0 \%$ and $30 \%$, with the average approximately $12 \%$, although making comparisons between published series is difficult because of the various methods and reporting criteria used by different authors. ${ }^{[33]}$ In the present study $4 \%$ (10/ $250)$ of patients had cerebrospinal fluid leak with 3 patients requiring surgical repair and the incidence is at par with the series published by Yamakani I et al, ${ }^{[14]}$ recently. However the incidence of associated meningitis in our study is slightly more $(8 \%)$ as compared to some of the recent series (between 3.7 to $9.2 \%)^{[13,17,18,22,34]}$

Although loss of cochlear and seventh nerve function are two of the major cranial nerves injuries that can occur during the surgery, there are risks of injury to lower cranial nerves in large and giant sized tumors, which can complicate the postoperative course. Judicious use of nasogastric tube feeding and planned tracheostomy can avoid major respiratory complications post-operatively. The incidence of lower cranial nerve paresis has been reported to range from $1.5 \%$ to $5.5 \%{ }^{[8,13,16,19]}$ against $6.8 \%$ in the present study.

In the present study, all the cases were operated by retrosigmoid approach with park-bench position, except in 2 patients with sitting position. This concludes the fact that the retro-sigmoid approach in experienced hands is a good option; with good results compared to other series irrespective of the tumor size. This is an extension to the view put forward by Samii et al that from any of the available approaches, such as the sub-occipital, the middle fossa, and the trans-labyrinthine; surgeons can develop expertise to high standards, by training and experience, with respect to the optimum patient's safety, mortality and morbidity. ${ }^{[16]}$

Gormley and Sekhar et al ${ }^{[9]}$ used the combined transpetrosal and retrosigmoid approach for tumors greater or more than 4 $\mathrm{cm}$ in the cerebello-pontine angle, especially when they extend up to the tentorial notch, because the combination allows good visualization of tumor-brain stem interface and the tentorial notch and better facial nerve outcome for these group of tumors. But in our series, using retro-sigmoid approach alone visualization of tumor-brainstem interface and facial nerve preservation could be possible in giant sized tumors with out much difficulty.

\section{References}

1. Balasubramaniam V, Ramamurthi B. Experience with auditory neurofibroma. India: Neurol; 1962;10:29.

2. Sambasivan M, Mathai KV, Chandy J. Surgical experience with eighty cases of acoustic neurinomas. India: Neurol; 1966;14:125

3. Ramamurthi B. Acoustic neurinomas in developing countries. International Symposium on Acoustic Neurinomas. India: Neurol; 1990;38:223.

4. Unni M, Rao VRK, Rout D, et al. Common CP angle tumors - A CT analysis. India: Neurol; 1991;39:117.

5. Ramamurthi B. The continuing challenge of acoustic neurinomas (1949-1993). Br J Neurosurg 1995;9:361-6. Review

6. House JW, Brackmann DE. Facial nerve grading system. Otolaryngol Head Neck Surg 1985;93:146-7

7. Samii M, Matthies C. Management of 1000 vestibular schwannomas (acoustic neuromas). Hearing function in 1000 tumor resections. Neurosurgery $1997 ; 40: 248-62$.

8. Jackler RK, Pitts LH. Acoustic Neuroma. Neurosurg Clin N Am 1990;1:199223.

9. Gormley WB, Sekhar LN, Wright DC, Kamerer D, Schessel D. Acoustic neuromas. Results of current surgical management. Neurosurgery $1997 ; 41: 50-60$

10. Ojemann RG, Martuza RL. Acoustic neuroma. Youmans JR, Neurological surgery, $3^{\text {rd }}$ Ed. Philadelphia: WB Saunders; 1990. p. 3316-50.

11. Rand RW, Kurze T. Facial nerve preservation by posterior fossa transmaetal microdissection in total removal of acoustic neuroma. J Neurol Neurosurg Psychiatry 1965;28:311-6.

12. Jung S, Kang SS, Kim TS, et al. Current surgical results of retrosigmoid approach in extralarge vestibular schwannoma. Surg Neurol 2000;53:370-7; discussion $377-8$.

13. Lanman TH, Brackmann DE, Hitselberger WE, Subin B. Report of 190 consecutive cases of large acoustic tumors (vestibular schwannoma) removed via the translabyrinthine approach. J Neurosurg 1999;90;617-23.

14. Yamakami I, Uchino Y, Kobayashi E, Yamaura A, Oka N. Removal of large acoustic neurinomas (vestibular schwannomas) by the retrosigmoid approach with no mortality and minimal morbidity. J Neurol Neurosurg Psychiatry $2004 ; 75: 453-8$

15. Ebersold MJ, Harner SG, Beatty CW, Harper CM, Quast LM. Current results of the retromastoid approach to acoustic neurinoma. J Neurosurg 1992;76:901-9

16. Samii M, Matthies C. Management of 1000 vestibular schwannomas (acoustic neuromas): Surgical management and results with an emphasis on complications and how to avoid them. Neurosurgery 1997;40:11-23.

17. Sluyter S, Graamans K, Tulleken CA, et al. Analysis of the results obtained in 120 patients with large acoustic neuromas surgically treated via the translybrinthine-transtentorial approach. J Neurosurg 2001;94:61-6.

18. Mamikoglu B, Weit RJ, Esquivel CR. Translabrynthine approach for the management of large and giant vestibular schwannomas. Otol Neurotol $2002 ; 23: 224-7$. 

approach to acoustic neurinomas. .J Neurosurg 1992;76:901-9. acoustic neuromas related to tumor size. Acta Otolaryngol Supp $1988 ; 452: 38-51$

21. Sterkers JM, Morrison GA, Sterkers O, El-Dine MM. Preservation of facial, cochlear, and other nerve functions in acoustic neuroma treatment. Otolaryngol Head Neck Surg 1994;110:146-55.

22. Briggs RJS, Luxford WM, Atkins JS, Hitselberger WE. Translabyrinthine removal of large acoustic neuromas. Neurosurgery 1994;34:785-92.

23. Samii M, Matthies C. Management of 1000 vestibular schwannomas (acoustic neuromas): The facial nerve -preservation and restitution of function. Neurosurgery 1997;40:684-95.

24. Whittaker CK, Luetje CM. Vestibular schwannomas. J Neurosurg 1992;76:897-900.

25. Cohen NL. Retrosigmoid approach for acoustic tumor removal. Otolaryngol Clin North Am 1992;25:295-310.

26. Fischer G, Fischer C, Remond J. Hearing preservation in acoustic neurinoma surgery. J Neurosurg 1992;76:910-7.

27. Haines S.J, Levine SC. Intracanalicular acoustic neuroma: Early surgery for preservation of hearing. J Neurosurg 1993;79:515-20.
29 Nadol JB Jr, Chiong CM, Ojemann RG, McKenna MJ, Martuza RL, Montgomery WW, et al. Preservation of hearing and facial nerve function in resection of acoustic neuroma. Laryngoscope 1992;102:1153-8.

30. Shelton C. Hearing preservation in acoustic tumor surgery. Otolaryngol Clin North Am 1992;25:609-21.

31. Slavit DH, Harner SG, Harper CM Jr, Bealty CW. Auditory monitoring during acoustic neuroma removal. Arch Otolaryngol Head Neck Surg 1991;117:1153-7.

32. Goel A, Sekhar LN, Langheinrich W, Kamerer D, Hirsch B. Late course of preserved hearing and tinnitus after acoustic neurlemoma surgery. J Neurosurg $1992 ; 77: 685-9$

33. Brennan JW, Rowed DW, Nedzelski JM, Chen JM. Cerebrospinal fluid leak after acoustic neuroma surgery: Influence of tumor size and surgical approach on incidence and response to treatment. J Neurosurg 2001;94:217-23.

34. Bryce GE, Nedzelski JM, Rowed DW, et al. Cerebrospinal fluid leaks and meningitis in acoustic neuroma surgery. Otolaryngol Head Neck Surg 1991;104:81-7

Accepted on 15.12.2004.

\section{Invited Comments}

The landscape of surgery for vestibular schwannomas is changing. Durable, consistently good outcomes have been achieved in patients with small-to-medium sized tumors treated with stereotactic radiosurgery. In addition, the "learning curve" effect on surgical outcomes has been documented to involve a greater number of cases than many previously thought, even in the hands of meticulous, dedicated surgeons. For this reason, many (if not most) patients with radiosurgically appropriate tumor volumes are being managed without surgery. At Penn State University, as in many other centers, vestibular schwannoma surgery is performed almost exclusively on patients with large tumor volumes who are not radiosurgical candidates.

It has long been recognized that tumor size is directly related to the risk of complications in VS surgery. Larger tumors are more likely to cause significant brainstem compression and the potential for decompression and dissection related brainstem injust if greater than with small tumors. Hearing and facial nerve function preservation are both inversely related to tumor size. In addition, blood loss and operation duration increase with larger tumors, both importing additional risk.

This article reports on the remarkable surgical results of a group of surgeons in India caring for patients with large vestibular schwannomas. The selection mechanisms for large tumors in this series are presumably more related to difficulty gaining access to medical care rather than radiosurgical bias. The authors clearly outline the patient population and discuss the causes for such large tumors and delayed medical attention. Aside from delayed diagnosis and healtheare access, these patients were treated in a fashion different from many centers elsewhere. The CUSA, routinely used in many centers for VS surgery, was rarely used. Facial nerve monitoring was not employed, and one assumes that more extensive monitoring was not used. In my practice, I have found neurophysiological monitoring to be of greater importance in resection of large tumors with significant brainstem compression. We routinely employ ABR, SSEP, and facial nerve monitoring on all VS cases. I have found these to be helpful when extensive brainstem compression exists pre-operatively, although certainly there is debate about the utility of such monitoring.

The authors are to be commended on their excellent surgical results overall and in particular in facial nerve preservation without monitoring. Many patients in this series had significant pre-operative deficits likely affecting overall health and perhaps contributing to the $6 \%$ mortality rate.

As patients with small/medium tumors are treated more frequently with non-operative techniques, vestibular schwannoma surgeons will find themselves facing larger tumors. The results of this study, along with other series of large tumors, confirm that although the risks are greater than for small tumors, large VS can be operated upon through the conventional suboccipital or translabyrinthine routes with acceptable results.

Jonas M. Sheehan

Department of Neurosurgery, Chief, Division of Neurooncology and Cranial Base Surgery, Penn State College of Medicine, Milton S. Hershey Medical Center, 500 University Drive, Hershey, PA, 17033, USA. E-mail: jsheehan@psu.edu 